Article

\title{
A Sharp Rellich Inequality on the Sphere
}

\section{Songting Yin ${ }^{1,2}$}

1 Department of Mathematics and Computer Science, Tongling University, Tongling 244000, China; yst419@163.com

2 Key Laboratory of Applied Mathematics (Putian University), Fujian Province University, Putian 351100, China

Received: 25 October 2018; Accepted: 26 November 2018; Published: 27 November 2018

Abstract: We obtain a Rellich type inequality on the sphere and give the corresponding best constant. The result complements some related inequalities in recent literatures.

Keywords: rellich inequality; sphere; sharp constant

MSC: Primary 26D10; Secondary 46E36

\section{Introduction}

The classical Rellich inequality states that [1], for $n \geq 5$ and all $f \in C_{c}^{\infty}\left(\mathbb{R}^{n} \backslash\{0\}\right)$,

$$
\int_{\mathbb{R}^{n}}|\Delta f|^{2} d x \geq \frac{n^{2}(n-4)^{2}}{16} \int_{\mathbb{R}^{n}} \frac{f^{2}}{|x|^{4}} d x
$$

The constant $\frac{n^{2}(n-4)^{2}}{16}$ is optimal and never archived. Under additional conditions there are also versions for lower dimensions. There has been a lot of research concerning the Rellich inequality on the Euclidean space due to its applications to spectral theory, harmonic analysis, geometry and partial differential equations. We see [2-6] and the references therein.

The validity of the Rellich inequality on a manifold and its best constants allows people to obtain qualitative properties on the manifold. For complete noncompact Riemannian manifolds, under some geometric assumptions on the weight function $\rho$, Kome and Özaydin [7] proved that for $f \in C_{c}^{\infty}\left(M-\rho^{-1}\{0\}\right)$ (where $\left.\alpha<2, C+\alpha-3>0\right)$

$$
\int_{M} \rho^{\alpha}|\Delta f|^{2} d V \geq \frac{(C+\alpha-3)^{2}(C-\alpha+1)^{2}}{16} \int_{M} \rho^{\alpha} \frac{f^{2}}{\rho^{4}} d V
$$

Particularly, they also obtained the improved versions of a Rellich-type inequality which involves both first and second order derivatives in the Poincaré conformal disc model $\left(n>2, \frac{8-n}{3}<\alpha<2\right)$

$$
\int_{\mathbb{B}^{n}} r^{\alpha}|\Delta f|^{2} d V \geq \frac{(n-\alpha)^{2}}{4} \int_{\mathbb{B}^{n}} r^{\alpha} \frac{|\nabla f|^{2}}{r^{4}} d V
$$

where $f \in C_{c}^{\infty}\left(\mathbb{B}^{n}\right)$ and $r=\log \frac{1+|x|}{1-|x|}$ is the geodesic distance. Furthermore, the constant $\frac{(n-\alpha)^{2}}{4}$ is sharp. Along this line, we refer to [4,7-11] and so on.

However, there are not many literatures discussing the Rellich inequality on the sphere so far. See [12-14] for details. In [14] Xiao derived the following inequality

$$
C \int_{\mathbb{S}^{n}} \frac{f^{2}}{\sin ^{2} d(p, x)} d V+\int_{\mathbb{S}^{n}}|\Delta f|^{2} d V \geq \frac{n^{2}(n-4)^{2}}{16}\left(\int_{\mathbb{S}^{n}} \frac{f^{2}}{d(p, x)^{4}} d V+\int_{\mathbb{S}^{n}} \frac{f^{2}}{(\pi-d(p, x))^{4}} d V\right)
$$


for $f \in C_{c}^{\infty}\left(\mathbb{S}^{n}-d^{-1}(0) \cup d^{-1}(\pi)\right)$, where $d(p, x)$ is the geodesic distance from $p$ to $x$ on $\mathbb{S}^{n}$ and $C$ is some positive constant. Moreover, the constant $\frac{n^{2}(n-4)^{2}}{16}$ is sharp.

In this short note we will obtain another type of Rellich inequality on the sphere and also give the corresponding sharp constant. Our main theorem is as follows:

Theorem 1. Let $\left(\mathbb{S}^{n}, g\right)(n \geq 5)$ be the $n$-sphere with sectional curvature 1 and $p$ be a fixed point in $\mathbb{S}^{n}$. Then for any function $f \in C_{c}^{\infty}\left(\mathbb{S}^{n}-d^{-1}(0) \cup d^{-1}(\pi)\right)$,

$$
C(n) \int_{\mathbb{S}^{n}} \frac{f^{2}}{\sin ^{2} d(p, x)} d V+\int_{\mathbb{S}^{n}}|\Delta f|^{2} d V \geq \frac{n^{2}(n-4)^{2}}{16} \int_{\mathbb{S}^{n}} \frac{f^{2}}{\sin ^{4} d(p, x)} d V,
$$

where $C(n)=\frac{n(n-4)\left(n^{2}-2 n-4\right)}{8}$ and the constant $\frac{n^{2}(n-4)^{2}}{16}$ is sharp.

Remark 1. In Euclidean spaces (resp. a Riemannian manifold, the Poincaré conformal disc model), the Laplacian of the distance function (resp. some weighted function) equals to $\frac{n-1}{|x|}$ (resp. is not less than $\frac{C}{\rho}, \frac{n-1}{r}$ ). Thus the Rellich inequality certainly contains the term $\frac{f^{2}}{|x|^{4}}\left(\right.$ resp. $\frac{f^{2}}{\rho^{4}}, \frac{f^{2}}{r^{4}}$ ). Since on the sphere the Laplacian of the distance function is $\Delta d(p, x)=(n-1) \cot d(p, x)$ when $d$ is smooth (see [15] p. 207), the terms $\frac{f^{2}}{\sin ^{2} d(p, x)}$ and $\frac{f^{2}}{\sin ^{4} d(p, x)}$ are naturally involved. So, it is a bit different in form from that in Euclidean spaces and some other type of Rellich inequalities. It is interesting that, even though the coefficient $C(n)$ is replaced by an arbitrary number, the constant $\frac{n^{2}(n-4)^{2}}{16}$ is still sharp. To prove the result, we give some modifications in constructing the auxiliary function, and then do calculations in two hemispheres by using the antipodal points. The remainder of the approaches used are similar to Xiao's paper [14]. See also in [7,16].

\section{The Proof of the Main Result}

Proof of Theorem 1. Denote by $r_{p}(x)=d(p, x)$ the distance function from the fixed point $p \in \mathbb{S}^{n}$. Let $f$ be a smooth function in $C_{c}^{\infty}\left(\mathbb{S}^{n} \backslash\{p, q\}\right)$, where $q$ is the antipodal point of $p$. Then

$$
\Delta f^{2}=2 f \Delta f+2|\nabla f|^{2},
$$

and thus

$$
-2 \int_{\mathbb{S}^{n}} \frac{f \Delta f}{\sin ^{2} r_{p}} d V=-\int_{\mathbb{S}^{n}} \frac{\Delta f^{2}}{\sin ^{2} r_{p}} d V+2 \int_{\mathbb{S}^{n}} \frac{|\nabla f|^{2}}{\sin ^{2} r_{p}} d V
$$

Compute

$$
\begin{aligned}
\int_{\mathbb{S}^{n}} \frac{\Delta f^{2}}{\sin ^{2} r_{p}} d V & =\int_{\mathbb{S}^{n}} f^{2} \Delta \sin ^{-2} r_{p} d V=\int_{\mathbb{S}^{n}} f^{2} \operatorname{div}\left(\nabla \sin ^{-2} r_{p}\right) d V \\
& =-2 \int_{\mathbb{S}^{n}} f^{2} \operatorname{div}\left(\sin ^{-3} r_{p} \cos r_{p} \nabla r_{p}\right) d V \\
& =-2 \int_{\mathbb{S}^{n}} f^{2}\left[\sin ^{-3} r_{p} \cos r_{p} \Delta r_{p}+\left(\sin ^{-3} r_{p} \cos r_{p}\right)^{\prime}\right] d V \\
& =-2 \int_{\mathbb{S}^{n}} f^{2}\left[\frac{n-4}{\sin ^{4} r_{p}}-\frac{n-3}{\sin ^{2} r_{p}}\right] d V,
\end{aligned}
$$

where we have used $\Delta r_{p}=(n-1) \cot r_{p}$ in the sphere. To estimate $\int_{\mathbb{S}^{n}} \frac{|\nabla f|^{2}}{\sin ^{2} r_{p}} d V$, we put $f=$ $\left(\sin r_{p}\right)^{-\frac{n-4}{2}} \varphi$. Then

$$
\nabla f=\varphi \nabla\left(\sin r_{p}\right)^{-\frac{n-4}{2}}+\left(\sin r_{p}\right)^{-\frac{n-4}{2}} \nabla \varphi,
$$


and

$$
\begin{aligned}
|\nabla f|^{2} & =\varphi^{2}\left|\nabla\left(\sin r_{p}\right)^{-\frac{n-4}{2}}\right|^{2}+\left(\sin r_{p}\right)^{-(n-4)}|\nabla \varphi|^{2}+2\left(\sin r_{p}\right)^{-\frac{n-4}{2}} \varphi\left\langle\nabla\left(\sin r_{p}\right)^{-\frac{n-4}{2}}, \nabla \varphi\right\rangle \\
& \geq \frac{(n-4)^{2}}{4} \varphi^{2}\left(\sin r_{p}\right)^{-(n-2)} \cos ^{2} r_{p}+\frac{1}{2}\left\langle\nabla\left(\sin r_{p}\right)^{-(n-4)}, \nabla \varphi^{2}\right\rangle .
\end{aligned}
$$

This gives

$$
\int_{\mathbb{S}^{n}} \frac{|\nabla f|^{2}}{\sin ^{2} r_{p}} d V \geq \frac{(n-4)^{2}}{4} \int_{\mathbb{S}^{n}} \frac{\varphi^{2}}{\left(\sin r_{p}\right)^{n}} \cos ^{2} r_{p} d V-\frac{n-4}{2(n-2)} \int_{\mathbb{S}^{n}} \varphi^{2} \Delta\left(\sin r_{p}\right)^{-(n-2)} d V .
$$

A direct computation shows that

$$
\begin{aligned}
\Delta\left(\sin r_{p}\right)^{-(n-2)} & =\operatorname{div}\left(\nabla\left(\sin r_{p}\right)^{-(n-2)}\right)=-(n-2) \operatorname{div}\left(\left(\sin r_{p}\right)^{-(n-1)} \cos r_{p} \nabla r_{p}\right) \\
& =-(n-2)\left(\sin r_{p}\right)^{-(n-1)} \cos r_{p} \Delta r_{p}-(n-2)\left(\left(\sin r_{p}\right)^{-(n-1)} \cos r_{p}\right)^{\prime} \\
& =(n-2)\left(\sin r_{p}\right)^{2-n} .
\end{aligned}
$$

Therefore,

$$
\begin{aligned}
\int_{\mathbb{S}^{n}} \frac{|\nabla f|^{2}}{\sin ^{2} r_{p}} d V & \geq \frac{(n-4)^{2}}{4} \int_{\mathbb{S}^{n}} \frac{\varphi^{2}}{\left(\sin r_{p}\right)^{n}} \cos ^{2} r_{p} d V-\frac{n-4}{2} \int_{\mathbb{S}^{n}} \varphi^{2}\left(\sin r_{p}\right)^{2-n} d V \\
& =\frac{(n-4)^{2}}{4} \int_{\mathbb{S}^{n}} \frac{\varphi^{2}}{\left(\sin r_{p}\right)^{n}} d V-\left[\frac{(n-4)^{2}}{4}+\frac{n-4}{2}\right] \int_{\mathbb{S}^{n}} \frac{\varphi^{2}}{\left(\sin r_{p}\right)^{n-2}} d V \\
& =\frac{(n-4)^{2}}{4} \int_{\mathbb{S}^{n}} \frac{f^{2}}{\sin ^{4} r_{p}} d V-\left[\frac{(n-4)^{2}}{4}+\frac{n-4}{2}\right] \int_{\mathbb{S}^{n}} \frac{f^{2}}{\sin ^{2} r_{p}} d V .
\end{aligned}
$$

By the Cauchy-Schwarz inequality, one has

$$
-2 \int_{\mathbb{S}^{n}} \frac{f \Delta f}{\sin ^{2} r_{p}} d V \leq \frac{n(n-4)}{4} \int_{\mathbb{S}^{n}} \frac{f^{2}}{\sin ^{4} r_{p}} d V+\frac{4}{n(n-4)} \int_{\mathbb{S}^{n}}|\Delta f|^{2} d V .
$$

Finally, combining (1) (4), we obtain

$$
\frac{n(n-4)\left(n^{2}-2 n-4\right)}{8} \int_{\mathbb{S}^{n}} \frac{f^{2}}{\sin ^{2} d(p, x)} d V+\int_{\mathbb{S}^{n}}|\Delta f|^{2} d V \geq \frac{n^{2}(n-4)^{2}}{16} \int_{\mathbb{S}^{n}} \frac{f^{2}}{\sin ^{4} d(p, x)} d V .
$$

In what follows, we show the constant $\frac{n^{2}(n-4)^{2}}{16}$ is sharp. The skill is borrowed from [16] (see also [14]). Let $\eta: R \rightarrow[0,1]$ be a smooth function such that $0 \leq \eta \leq 1$ and

$$
\eta(t)= \begin{cases}1, & t \in[-1,1] \\ 0, & |t| \geq 2\end{cases}
$$

Let $H(t)=1-\eta(t)$. For sufficient small $\varepsilon>0$, Set

$$
f_{\varepsilon}\left(r_{p}\right)= \begin{cases}0, & r_{p}=0 \\ H\left(\frac{r_{p}}{\varepsilon}\right) \sin ^{\frac{2-n}{2}} r_{p}, & 0<r_{p} \leq \frac{\pi}{2} \\ H\left(\frac{\pi-r_{p}}{\varepsilon}\right) \sin ^{\frac{2-n}{2}}\left(\pi-r_{p}\right), & \frac{\pi}{2} \leq r_{p}<\pi \\ 0, & r_{p}=\pi\end{cases}
$$

Observe that $f_{\varepsilon}\left(r_{p}\right)$ can be approximated by smooth functions on the sphere $\mathbb{S}^{n}$. 
Let $q$ be the antipodal point of $p$. Then $d(p, q)=\pi$ and for any point $x \in \mathbb{S}^{n}$ we have $r_{p}+r_{q}=\pi$. Since the constructed function $f_{\varepsilon}$ possesses a fair degree of bilateral symmetry on the sphere, it is easier to compute in the following by using the antipodal points $p$ and $q$.

$$
\begin{aligned}
\int_{\mathbb{S}^{n}} \frac{f_{\varepsilon}^{2}}{\sin ^{2} r_{p}} d V & =\int_{B_{p}\left(\frac{\pi}{2}\right)} \frac{f_{\varepsilon}^{2}}{\sin ^{2} r_{p}} d V+\int_{B_{q}\left(\frac{\pi}{2}\right)} \frac{f_{\varepsilon}^{2}}{\sin ^{2} r_{p}} d V \\
& =\operatorname{Vol}\left(\mathbb{S}^{n-1}\right) \int_{\varepsilon}^{\frac{\pi}{2}} H^{2}\left(\frac{r_{p}}{\varepsilon}\right) \sin r_{p} d r+\operatorname{Vol}\left(\mathbb{S}^{n-1}\right) \int_{\frac{\pi}{2}}^{\pi-\varepsilon} H^{2}\left(\frac{\pi-r_{p}}{\varepsilon}\right) \sin \left(\pi-r_{p}\right) d r \\
& =2 \operatorname{Vol}\left(\mathbb{S}^{n-1}\right) \int_{\varepsilon}^{\frac{\pi}{2}} H^{2}\left(\frac{r_{p}}{\varepsilon}\right) \sin r_{p} d r \\
& \leq \pi \operatorname{Vol}\left(\mathbb{S}^{n-1}\right), \\
\int_{\mathbb{S}^{n}} \frac{f_{\varepsilon}^{2}}{\sin ^{4} r_{p}} d V= & \int_{B_{p}\left(\frac{\pi}{2}\right)} \frac{f_{\varepsilon}^{2}}{\sin ^{4} r_{p}} d V+\int_{B_{q}\left(\frac{\pi}{2}\right)} \frac{f_{\varepsilon}^{2}}{\sin ^{4} r_{p}} d V \\
= & \operatorname{Vol}\left(\mathbb{S}^{n-1}\right) \int_{\varepsilon}^{\frac{\pi}{2}} H^{2}\left(\frac{r_{p}}{\varepsilon}\right) \sin ^{-1} r_{p} d r+\operatorname{Vol}\left(\mathbb{S}^{n-1}\right) \int_{\frac{\pi}{2}}^{\pi-\varepsilon} H^{2}\left(\frac{\pi-r_{p}}{\varepsilon}\right) \sin ^{-1}\left(\pi-r_{p}\right) d r \\
& =2 \operatorname{Vol}\left(\mathbb{S}^{n-1}\right) \int_{\varepsilon}^{\frac{\pi}{2}} H^{2}\left(\frac{r_{p}}{\varepsilon}\right) \sin ^{-1} r_{p} d r \\
& \geq 2 \operatorname{Vol}\left(\mathbb{S}^{n-1}\right) \int_{2 \varepsilon}^{\frac{\pi}{2}} \sin ^{-1} r_{p} d r .
\end{aligned}
$$
and thus

Next we are to estimate $\int_{\mathbb{S}^{n}}\left|\Delta f_{\varepsilon}\right|^{2} d V$. When $0<r_{p}<\frac{\pi}{2}$, the distance function $d(p, x)$ is smooth,

$$
\begin{aligned}
\Delta f_{\varepsilon} & =\operatorname{div}\left(\nabla f_{\varepsilon}\right) \\
& =\operatorname{div}\left(\left(\frac{1}{\varepsilon} H^{\prime}\left(\frac{r_{p}}{\varepsilon}\right)(\sin r)^{-\frac{n-4}{2}}-\frac{n-4}{2} H\left(\frac{r_{p}}{\varepsilon}\right)\left(\sin r_{p}\right)^{-\frac{n-2}{2}} \cos r_{p}\right) \nabla r_{p}\right) \\
= & \left(\frac{1}{\varepsilon} H^{\prime}\left(\frac{r_{p}}{\varepsilon}\right)\left(\sin r_{p}\right)^{-\frac{n-4}{2}}-\frac{n-4}{2} H\left(\frac{r_{p}}{\varepsilon}\right)\left(\sin r_{p}\right)^{-\frac{n-2}{2}} \cos r_{p}\right)(n-1) \cot r_{p} \\
& +\left(\frac{1}{\varepsilon} H^{\prime}\left(\frac{r_{p}}{\varepsilon}\right)\left(\sin r_{p}\right)^{-\frac{n-4}{2}}-\frac{n-4}{2} H\left(\frac{r_{p}}{\varepsilon}\right)\left(\sin r_{p}\right)^{-\frac{n-2}{2}} \cos r_{p}\right)^{\prime} \\
= & \frac{1}{\varepsilon^{2}}\left(\sin r_{p}\right)^{-\frac{n-4}{2}} H^{\prime \prime}\left(\frac{r_{p}}{\varepsilon}\right)+\frac{3}{\varepsilon}\left(\sin r_{p}\right)^{-\frac{n-2}{2}} \cos r_{p} H^{\prime}\left(\frac{r_{p}}{\varepsilon}\right) \\
& -\frac{n-4}{2} H\left(\frac{r_{p}}{\varepsilon}\right)\left[\frac{n}{2}\left(\sin r_{p}\right)^{-\frac{n}{2}} \cos ^{2} r_{p}-\left(\sin r_{p}\right)^{-\frac{n-4}{2}}\right],
\end{aligned}
$$

and when $\frac{\pi}{2}<r_{p}<\pi$, one can get the same formula as above by letting $r_{q}=\pi-r_{p}$. Therefore,

$$
\int_{\mathbb{S}^{n}}\left|\Delta f_{\varepsilon}\right|^{2} d V=\int_{B_{p}\left(\frac{\pi}{2}\right)}\left|\Delta f_{\varepsilon}\right|^{2} d V+\int_{B_{q}\left(\frac{\pi}{2}\right)}\left|\Delta f_{\varepsilon}\right|^{2} d V=2 \int_{B_{p}\left(\frac{\pi}{2}\right)}\left|\Delta f_{\varepsilon}\right|^{2} d V
$$


and thus by Minkowski inequality,

$$
\begin{aligned}
& \frac{1}{\operatorname{Vol}\left(\mathbb{S}^{n-1}\right)^{\frac{1}{2}}}\left(\int_{\mathbb{S}^{n}}\left|\Delta f_{\varepsilon}\right|^{2} d V\right)^{\frac{1}{2}} \\
= & \frac{\sqrt{2}}{\operatorname{Vol}\left(\mathbb{S}^{n-1}\right)^{\frac{1}{2}}}\left(\int_{B_{p}\left(\frac{\pi}{2}\right)}\left|\Delta f_{\varepsilon}\right|^{2} d V\right)^{\frac{1}{2}} \\
\leq & \sqrt{2}\left(\int_{\mathcal{E}}^{\frac{\pi}{2}} H^{2}\left(\frac{r_{p}}{\varepsilon}\right)\left|\frac{-n(n-4)}{4}\left(\sin r_{p}\right)^{-\frac{n}{2}} \cos ^{2} r_{p}+\frac{n-4}{2}\left(\sin r_{p}\right)^{-\frac{n-4}{2}}\right|^{2}\left(\sin r_{p}\right)^{n-1} d r\right)^{\frac{1}{2}} \\
& +\frac{3 \sqrt{2}}{\varepsilon}\left(\int_{\varepsilon}^{2 \varepsilon}\left|\left(\sin r_{p}\right)^{-\frac{n-2}{2}} \cos r_{p} H^{\prime}\left(\frac{r_{p}}{\varepsilon}\right)\right|^{2}\left(\sin r_{p}\right)^{n-1} d r\right)^{\frac{1}{2}} \\
& +\frac{\sqrt{2}}{\varepsilon^{2}}\left(\int_{\varepsilon}^{2 \varepsilon}\left|\left(\sin r_{p}\right)^{-\frac{n-4}{2}} H^{\prime \prime}\left(\frac{r_{p}}{\varepsilon}\right)\right|^{2}\left(\sin r_{p}\right)^{n-1} d r\right)^{\frac{1}{2}} \\
= & I+I I+I I I .
\end{aligned}
$$

A straightforward calculation yields

$$
\begin{aligned}
I & \leq \sqrt{2}\left(\int_{\varepsilon}^{\frac{\pi}{2}}\left|\frac{-n(n-4)}{4}\left(\sin r_{p}\right)^{-\frac{n}{2}}+\frac{(n-4)(n+2)}{4}\left(\sin r_{p}\right)^{-\frac{n-4}{2}}\right|^{2}\left(\sin r_{p}\right)^{n-1} d r\right)^{\frac{1}{2}}, \\
I I & \leq \frac{3 \sqrt{2}}{\varepsilon} \max _{t \in[0,2]} H^{\prime}(t)\left(\int_{\varepsilon}^{2 \varepsilon}\left|\left(\sin r_{p}\right)^{-\frac{n-2}{2}} \cos r_{p}\right|^{2}\left(\sin r_{p}\right)^{n-1} d r\right)^{\frac{1}{2}} \\
& \leq \frac{3 \sqrt{2}}{\varepsilon} \max _{t \in[0,2]} H^{\prime}(t)\left(\int_{\varepsilon}^{2 \varepsilon} r_{p} d r\right)^{\frac{1}{2}}=3 \sqrt{3} \max _{t \in[0,2]} H^{\prime}(t), \\
I I I & \leq \frac{2}{\varepsilon^{2}} \max _{t \in[0,2]} H^{\prime \prime}(t)\left(\int_{\varepsilon}^{2 \varepsilon} \sin ^{3} r_{p} d r\right)^{\frac{1}{2}} \leq \frac{2}{\varepsilon^{2}} \max _{t \in[0,2]} H^{\prime \prime}(t)\left(\int_{\varepsilon}^{2 \varepsilon} r_{p}^{3} d r\right)^{\frac{1}{2}}=\sqrt{15} \max _{t \in[0,2]} H^{\prime \prime}(t) .
\end{aligned}
$$

Since $f_{\varepsilon}\left(r_{p}\right)$ can be approximated by smooth functions on the sphere $\mathbb{S}^{n}$, then, by (5)-(7), it holds that

$$
\begin{aligned}
& \inf _{f \in C^{\infty}\left(\mathbb{S}^{n}\right) \backslash\{0\}} \frac{\int_{\mathbb{S}^{n}}|\Delta f|^{2} d V+C(n) \int_{\mathbb{S}^{n}} \frac{f^{2}}{\sin ^{2} r_{p}} d V}{\int_{\mathbb{S}^{n}} \frac{f^{2}}{\sin ^{4} r_{p}} d V} \\
\leq & \frac{\int_{\mathbb{S}^{n}}\left|\Delta f_{\varepsilon}\right|^{2} d V+C(n) \int_{\mathbb{S}^{n}} \frac{f_{\varepsilon}^{2}}{\sin ^{2} r_{p}} d V}{\int_{\mathbb{S}^{n}} \frac{f_{\varepsilon}^{2}}{\sin ^{4} r_{p}} d V} \\
\leq & \frac{\pi C(n)}{2 \int_{2 \varepsilon}^{\frac{\pi}{2}} \sin ^{-1} r_{p} d r}+\left(\frac{I+I I+I I I}{\sqrt{2} \sqrt{\int_{2 \varepsilon}^{\frac{\pi}{2}} \sin ^{-1} r_{p} d r}}\right)^{2} .
\end{aligned}
$$

Letting $\varepsilon \rightarrow 0^{+}$, we have 


$$
\begin{aligned}
& \inf _{f \in C^{\infty}\left(\mathbb{S}^{n}\right) \backslash\{0\}} \frac{\int_{\mathbb{S}^{n}}|\Delta f|^{2} d V+C(n) \int_{\mathbb{S}^{n}} \frac{f^{2}}{\sin ^{2} r_{p}} d V}{\int_{\mathbb{S}^{n}} \frac{f^{2}}{\sin ^{4} r_{p}} d V} \\
\leq & \lim _{\varepsilon \rightarrow 0^{+}} \frac{\int_{\varepsilon}^{\frac{\pi}{2}}\left|\frac{-n(n-4)}{4}\left(\sin r_{p}\right)^{-\frac{n}{2}}+\frac{(n-4)(n+2)}{4}\left(\sin r_{p}\right)^{-\frac{n-4}{2}}\right|^{2}\left(\sin r_{p}\right)^{n-1} d r}{\int_{2 \varepsilon}^{\frac{\pi}{2}} \sin ^{-1} r_{p} d r} \\
= & \lim _{\varepsilon \rightarrow 0^{+}} \frac{-\left|\frac{-n(n-4)}{4}(\sin \varepsilon)^{-\frac{n}{2}}+\frac{(n-4)(n+2)}{4}(\sin \varepsilon)^{-\frac{n-4}{2}}\right|^{2}(\sin \varepsilon)^{n-1}}{-2 \sin (2 \varepsilon)^{-1}} \\
= & \frac{n^{2}(n-4)^{2}}{16} .
\end{aligned}
$$

This completes the proof.

Funding: This project is supported by AHNSF (1608085MA03), NNSFC(11471246) and KLAMFJPU (No. SX201805).

Conflicts of Interest: The authors declare no conflict of interest.

\section{References}

1. Rellich, F. Perturbation Theory of Eigenvalue Problems; Gordon and Breach: New York, NY, USA, 1969.

2. Brezis, H.; Vázquez, J.L. Blow-up solutions of some nonlinear elliptic problems. Rev. Mat. Univ. Comp. Madrid 1997, 10, 443-469.

3. Costa, D.G. On Hardy-Rellich type inequalities in $\mathbb{R}^{n}$. Appl. Math. Lett. 2009, 22, 902-905. [CrossRef]

4. Davies, E.B.; Hinz, A.M. Explicit constants for Rellich inequalities in $L_{p}(\Omega)$. Math. Z. 1998, 227, 511-523. [CrossRef]

5. Gazzola, F.; Grunau, H.-C.; Mitidieri, E. Hardy inequalities with optimal constants and remainder terms. Trans. Am. Math. Soc. 2004, 356, 2149-2168. [CrossRef]

6. Tertikas, A.; Zographopoulos, N. Best constants in the Hardy-Rellich Inequalities and Related Improvements. Adv. Math. 2007, 209, 407-459. [CrossRef]

7. Kombe, I.; Özaydin, M. Improved Hardy and Rellich inequalities on Riemannian manifolds. Trans. Am. Math. Soc. 2009, 361, 6191-6203. [CrossRef]

8. Grillo, G. Hardy and Rellich-type inequalities for metrics defined by vector fields. Potential Anal. 2003, 18, 187-217. [CrossRef]

9. Ghoussoub, N.; Moradifam, A. Bessel pairs and optimal Hardy and Hardy-Rellich inequalities. Math. Ann. 2011, 349, 1-57. [CrossRef]

10. Kombe, I.; Özaydin, M. Hardy-Poincaré, Rellich and uncertainty principle inequalities on Riemannian manifolds. Trans. Am. Math. Soc. 2013, 365, 5035-5050. [CrossRef]

11. Du, F.; Mao, J. Hardy and Rellich type inequalities on metric measure spaces. J. Math. Anal. Appl. 2015, 429, 354-365. [CrossRef]

12. Abolarinwa, A.; Apata, T. $L^{p}$-Hardy-Rellich and uncertainty principle inequalities on the sphere. Adv. Oper. Theory 2018, 3, 745-762. [CrossRef]

13. Dai, F.; Xu, Y. The Hardy-Rellich Inequality and Uncertainty Principle Inequalities on the Sphere. Constr. Approx. 2014, 40, 141-171. [CrossRef]

14. Xiao, Y. Some Hardy inequalities on the sphere. J. Math. Inequal. 2016, 10, 793-805. [CrossRef]

15. Bai, Z.; Shen, Y.; Shui, N.; Guo, X. An introduction to Riemann Geometry; Higher Education Press: Beijing, China, 2004; Volume 12.

16. Yang, Q.; Su, D.; Kong, Y. Hardy inequalities on Riemannian manifolds with negative curvature. Commun. Contemp. Math. 2014, 16, 1350043. [CrossRef] 